\section{Search for natural isotope superlattices}

SiR-The search for the formation of superlattice-type structures has yielded a good harvest for condensed matter scientists. Period doublings, commensurateincommensurate transitions, spontaneous ordering of voids in metals under neutron irradiation, and so forth, all represent examples of self-organization (orderdisorder transitions) in non-equilibrium systems in the sense of Prigogine synergetics ${ }^{1}$. In all cases of self-ordering two requirements are important: first, there should be some interaction between ordering species distinct from the host interactions; second, the ordered state of the minority species (voids among metal atoms, electrons forming Wigner lattice, and so on) should correspond to the minimum energy configuration.

Here I would like to indicate another probable, though not yet observed, candidate for the superlattice club - namely, the isotope superlattices (ISL). The interaction between atoms of different isotopes is not exactly the same ${ }^{2}$. Some small (about 1 part in 1,000) differences in lattice constants of isotopically pure crystals (for example, ${ }^{28} \mathrm{Si}$ and ${ }^{30} \mathrm{Si}$ ) arise because of the anharmonicity of zero-point vibrations. In an isotopically mixed crystal this could lead to a 'net' isotope interaction between atoms of the same isotope that is roughly the difference between the interaction of different isotope pairs. This will not always lead to the ordering tendency - it will depend on the particular isotope ratio. But in many cases the ordered arrangement of the minority isotope will be energetically preferable.

It is, of course, highly improbable that any natural isotope ratios will be such that the formation of ISL will occur without stoichiometric mismatch. Also, the required kinetics of crystallization should fall into quite specific margins. This probably explains why ISL have not yet been detected in natural samples. However the required isotope ratios can easily be obtained experimentally. Various solidifying liquids, such as mixtures of light and heavy water, combined with various external conditions, such as irradiation or external fields, provide an enormous variety of experimental opportunities. Although interesting in its own right, the search for isotope correlations and ISL (both natural and artificial) could also have many applications. I have recently discussed $^{3}$ the possibility of information storage based on isotope combinations.

Alexander A. Berezin

Department of Engineering Physics, McMaster University, Hamilton, Ontario, Canada, L8S 4M1

1. Woo, C. H. \& Frank, W. J. nucl. Mater. 137, 7-21 (1985)

2. Berezin, A. A. Chem. Phys. Lett. 110, 385-387 (1984).

3. Berezin, A. A. Kybernetes 15, 15-18 (1986).

\section{Amorphous definitions and glasses}

SIR-In their recent paper Phillips et al. present evidence for the existence of supernanometric microcystallites in 'amorphous' silicon. We should admit, however, that there are many ways in which a solid does not have to be a crystal: the random network model of the structure of glasses does not always give way to clusters of microcrystallites.

There is incontrovertible evidence that quartz glass has a coherence length of about $1 \mathrm{~nm}$, as expected for a random network: the thermal phonon ${ }^{2}$ mean free path at $80 \mathrm{~K}$ is only $1.2 \mathrm{~nm}$, as compared with 70 $\mathrm{nm}$ in quartz crystal (parallel to the $c$ axis). There is no elastic scattering process known to me that can reduce a phonon to such a short path except phase cancellation between atomic motions on adjacent arms of a random network, by which is meant that a phonon moving from atom $\mathrm{A}$ to atom $E$ proceeds in part by a long path $\mathrm{ABDE}$ and in part by a short path $\mathrm{ACE}$. Between room temperature and $100{ }^{\circ} \mathrm{C}$ the mean free path in the glass is about 0.8 $\mathrm{nm}$, consistent with structural coherence

\section{Lung surfactant anchor}

SIR-Patthy's' recent correspondence regarding the nature of the protein component of pulmonary surfactant is intriguing. As he points out, the major protein in both human and canine lung surfactant seems to have a collagen-like region within its amino-terminal half and a noncollagenous globular domain within its carboxy-terminal moiety. Of particular interest is the fact that the non-collagenous domain strongly resembles a module that is present in the proteoglycan of rat chondrosarcoma, the humoral lectin of the insect, Sarcophaga peregrina, and the membrane-associated lectins of human, rat and chicken hepatocytes.

In a recent cytochemical study of human neonatal lungs ${ }^{2}$, I found that the filamentous surface coating of the epithelial cells ('pneumonocytes') in the distal air spaces had binding sites for several lectins suggesting that the following carbohydrate groups are present in the filamentous material: $N$-acetylgalactosamine, $N$-acetylglucosamine, $\quad \alpha$-D-mannose, $\beta$-D-galactose and sialic acid. Another study ${ }^{3}$ indicates that the surface coating of hamster pneumonocytes and alveolar macrophages also contains a similar range of terminal carbohydrate groups.

It is generally assumed that pulmonary surfactant is closely associated with the surface of the respiratory epithelium. The popular view, originally advanced by Pattle $^{4}$, is that surfactant molecules form a monolayer at the interface between the alveolar air and the thin layer of fluid that moistens the epithelial cells. As the lectin of the same dimension. A structure of 3$\mathrm{nm}$ microcrystallites would give proportionately longer mean free paths near room temperature, and the increase at low temperatures as we enter the Rayleigh region would be deferred to still lower temperatures.

The enormous photon extinction length of transmission-quality optical fibres is consistent with a random network model of the glasses used in the fibres. Rayleigh scattering ${ }^{3}$ in a wide frequency region is the dominant intrinsic mechanism that limits the extinction, and here we are talking about propagation over 100 $\mathrm{km}$. Rayleigh scattering is proportional to the coherence volume: a change from $(0.8$ $\mathrm{nm})^{3}$ to $(3 \mathrm{~nm})^{3}$ would have a measurable deleterious effect.

Department of Physics,

University of California,

Berkeley, California 94720 ,

USA

1. Phillips, J.C., Bean, J.C., Wilson, B.A. \& Ourmazd, A. Nature 325, 121-125 (1987)

2. Kittel, C. Phys. Rev. 75, $972-974$ (1949)

3. Landau, L. \& Lifshitz, E. Electrodynamics of Continuous Media Ch. 14 (Pergamon, Oxford, 1960).

component of lung surfactant is similar to that derived from hepatocyte membranes and cartilage proteoglycans, it can be expected to be capable of binding to the terminal $N$-acetylglucosamine groups in the surface coating of the alveolar pneumonocytes. The extension of the filaments into the fluid subphase of the surfactant monolayer would facilitate the binding process.

Patthy ${ }^{1}$ has raised the possibility that the lectin-like component of surfactant protein interacts with the carbohydrate constituents of the lung surfactant complex. I share this opinion and, further, suggest that carbohydrate-lectin binding may help anchor the delicate surfactant film to the surface of the respiratory epithelium.

Department of Anatomy,

Cowan Meban

The Queen's University of Belfast, Belfast BT9 $7 B L$,

Northern Ireland

Patthy, L. Nature 325, 490 (1987)

2. Meban, C. Histochem. J. 18, 196-202 (1986)

3. Meban, C. J. Anat. 146, 131-139 (1986)

4. Pattle, R. E. Nature 175, 1125-1126 (1955).

\section{Scientific Correspondence}

Scientific Correspondence is intended to provide a forum in which readers may raise points of a scientific character. They need not arise out of anything published in Nature. In any case, priority will be given to letters of less than 500 words and five references. 